\title{
A COMMON IMAGE REPRESENTATION AND A PATCH-BASED SEARCH FOR CORRELATIVE LIGHT-ELECTRON-MICROSCOPY (CLEM) REGISTRATION
}

\author{
Bertha Mayela Toledo Acosta*, Patrick Bouthemy and Charles Kervrann \\ Inria, Centre Rennes - Bretagne Atlantique, Campus de Beaulieu, 35042 Rennes cedex, France
}

\begin{abstract}
Correlative light-electron microscopy (CLEM) enables to relate dynamics (or functions) with structure for a better understanding of cell mechanisms. However, the LM and EM images are of very different size, spatial resolution, field of view, and appearance. Registration of LM and EM modalities is then a timely, important but difficult open problem, which still requires some manual assistance. We have designed an original automated CLEM retracing-and-registration method involving a common representation with an adaptive associated scale (or blurring), the determination of the EM patch geometry, and the specification of appropriate descriptors and similarity criterion for the EM patch search. Its efficiency is demonstrated on real CLEM images.
\end{abstract}

Index Terms - CLEM, LoG transform, histogram distance, patch search, image registration.

\section{Introduction}

Light microscopy (LM) and electron microscopy (EM) are two fundamental investigation tools in cell biology. LM imaging allows the visualization of live cellular and subcellular behaviours but at relatively low spatial resolution. Different probes, dyes, fluorescent labels can be used to make the elements of interest visible. Phase-contrast LM images can also be used. In contrast, EM imaging enables the examination of the whole cellular ultrastructure at very high spatial resolution, but at the cost of fixating or freezing the cell. Correlative light-electron microscopy (CLEM) is then of key interest to combine the respective complementary strengths of LM and EM. However, managing to do it is not that straightforward. In recent years, research has been active in biology and microscopy to design efficient setups and protocols to achieve consecutive LM and EM acquisitions of the same sample [2, 5, 7, 13, 14, 15].

Broadly speaking, the usual workflow is to first acquire a LM image sequence of the living cell, to detect and locate the event of interest, then to chemically fixate or to freeze the sample and to acquire the EM image. Several variants

*This work has been partly supported by the National Council of Science and Technology of Mexico (CONACYT) under the thesis grant no. $246372 / 384571$. exist depending on the specific LM and EM modalities, the sample preparation (used probes), the image dimensions (2D or 3D), the overall setup to link up LM and EM acquisitions. The overall goal of CLEM is to relate dynamics (or functions) with structure for a better understanding of cell mechanisms.

Registering LM and EM images is then a key issue, but stands as a very difficult problem due to the big differences in spatial resolution, field of view and appearance. It usually requires manual interventions and the use of artificial landmarks (fiducial markers as beads, marker grids) at different stages of the overall CLEM workflow. The foreseen generalization of CLEM setups and the increasing mass of produced data impel to develop more automated workflows [8].

In this paper, we present an original automated approach to search for the EM patch corresponding to the LM region of interest (ROI) and to register them. It involves a common representation for the LM and EM images with an adaptive associated scale (or blurring), the determination of the EM patch geometry, and appropriate descriptors and similarity criterion for the EM patch search. The paper is organized as follows. Section 2 is devoted to related work. We describe our CLEM registration method in Section 3. In Section 4, we report experimental results on two sets of real CLEM images, and we give concluding comments in Section 5.

\section{Related Work}

A CLEM experiment involves among others two important issues: guidance of EM image acquisition from the LM image, and registration of LM and EM images [2]. Here, we are dealing with the second stage. The first stage aims at defining where the EM acquisition should be performed in the sample. The second stage can be further subdivided in the matching (or pairing, or retracing) step and the registration computation to overlay both images. Usually, manual interventions occur in these two stages, with the help of grids marked on the sample holder or the coverslip, and fiducial markers added in the sample.

The signals of fluospheres in the LM image are identified and manually assigned to the corresponding points in the averaged EM images in [10]. The coordinates of the pairs are then used as landmarks for generating linear conformal transformations relating the LM image with the EM image. In 
[14], EM images are relocated in LM images using two procedures. Larger structures are retrieved manually by a visual comparison of the LM scan with the overview scan of EM. For smaller structures, three reference points are chosen, and with a coordinate transformation the marked positions on the LM scan can be retrieved in the EM map. A similar approach was adopted in [6].

Very few investigations have been undertaken so far from an image processing perspective to make the registration more automatic. In [13], the image superposition is achieved by exploiting the auto-fluorescence which exhibits strong enough contours. Following a template-based approach, affine transformations are then applied until the one supplying the highest cross-correlation score is found, first with an EM image at low magnification, then for progressively higher magnifications. In [11], a method for feature-based registration is described, which exploits cell centroids and possibly nucleus. Yet, the registration accuracy heavily depends on the presence of sufficiently salient structures and their correct segmentation. A very different approach is proposed in [3]. It aims at transforming the two-modality issue into a single modality image registration problem. They introduce the so-called paradigm of image analogies and exploit a sparse representation model to obtain these image analogies. Convincing results are reported but this scheme requires a prior supervised learning stage which is not always affordable.

\section{Automated CLEM Registration}

We propose a novel method to achieve automatic pairing and registration between LM and EM images. It is able to manage significant differences in appearance, field of view, spatial resolution and content scale between both modalities. The overall workflow is divided into three main steps: 1) Common LoG representation for 2D EM and LM images with scale adaptation; 2) Fixing the EM patch geometry and seeking LM-ROI in EM; 3) Registering LM and EM patches and overlaying EM image onto LM image around ROI.

\subsection{Common LOG representation for EM and LM}

The idea is to find a transformation of both 2D LM and EM images which produces images of closer appearance. The Laplacian of Gaussian (LoG) transform is a good candidate, since it suppresses local linear intensity variations and enhances high spatial frequencies, while being linear, simple to apply, producing scalar values and enabling scale adaptation. The LoG transform of an image $I$ can be written as follows:

$$
\mathscr{L}_{\sigma}(I)=\Delta\left(G_{\sigma} * I\right)
$$

To compute the LoG of LM and EM images and select the right scales $s_{L M}$ and $s_{E M}$ given by the Gaussian variance $\sigma^{2}$, we use the first stage of our spot detection method ATLAS [1]. ATLAS automatically detects the characteristic scale of the objects of interest, by exploiting the Lindeberg's scale-space approach. The selected scale $\hat{\sigma}^{2}$ is the one with the maximum number of blobs, a blob being defined as a local minimum in the constructed scale-space domain. The ability of fixing $\hat{\sigma}^{2}$ values adapted to the LM and EM content respectively, will allow us to mitigate the difference in content scale of LM and EM images. The selected scale is expected to be higher for the EM image, yielding a stronger blurring of the EM image.

To match the LoG-LM ROI with the right patch in the LoG-EM image, we need first to delineate the ROI in the LoG-LM image. This ROI is supposed to encompass the dynamic event of interest, only observable through LM. In a given application, such an event could be automatically detected in the LM image sequence, using an appropiate method. Here, we assume that the ROI is already available.

\subsection{Retracing LoG-LM-ROI in LoG-EM}

To automatically retrace the LoG-LM-ROI in the LoGEM image, we carry out a patch-based exhaustive search. There is a considerable transformation (combination of rotation, shift, magnification,...) between LM and EM images. Hence, we resort to histograms that are invariant to rotation and scale. We implement two histogram-based methods to measure the similarity between a patch in the LoG-EM image and the LoG-LM-ROI. The first one involves LoG-value histograms and the second one exploits Local Directional Pattern (LDP)[9]. We tested several histogram distances to measure the similarity between LoG-LM-ROI and LoG-EM patch: KL-divergence, Jeffrey-divergence, cosine similarity, histogram intersection, $\chi^{2}$ distance, Bhattacharyya distance and Kolmogorov-Smirnov distance [4]. The best resuts were obtained with the cosine similarity and the histogram intersection, respectively defined by:

$$
\begin{aligned}
d_{c o s}\left(H_{L M}, H_{E M}\right) & =\frac{\sum_{v=1}^{V} H_{L M}(v) H_{E M}(v)}{\sqrt{\sum_{v=1}^{V} H_{L M}^{2}(v)} \sqrt{\sum_{v=1}^{V} H_{E M}^{2}(v)}}, \\
d_{h i}\left(H_{L M}, H_{E M}\right) & =\sum_{v=1}^{V} \min \left(H_{L M}(v), H_{E M}(v)\right),
\end{aligned}
$$

where $H_{L M}$, resp. $H_{E M}$, is the histogram computed over the LoG-LM ROI, resp. on the LoG-EM patch, $v$ denotes the histogram bin and $V$ the total number of bins. Histogram values are normalized over $[0,1]$.

Histograms of LoG values can be computed as explained above. A more elaborate descriptor may be necessary to capture more information. LDP is a local descriptor, used primarily for recognizing human faces [9]. It creates a pattern from an eight-bit binary code at each pixel of the input image I. Using Kirsch masks, the code is calculated by comparing the edge response values of a pixel in eight different orientations. The pattern records only the $k$ most significant directions (with the cooresponding bits) and the rest of the bits are set to 0 . With $k=3$, the LDP may generate up to 56 distinct 
patterns, creating an encoded image $\mathscr{D}_{I}$. We apply this operation to the LoG-LM ROI and the LoG-EM patch, producing encoded subimages, respectively denoted $\mathscr{D}_{L M}$ and $\mathscr{D}_{E M}$, and histograms of these values are computed. We use the same histogram distances as in (2).

Since no a priori information is available on the possible location of the ROI in the EM image and the transformation of the ROI location between EM and LM can be considerable, there is no immediate way to reduce the search space. We implement a patch-based exhaustive search to explore all potential locations in the LoG EM image. To specify the patch to be searched in the LoG EM image, we start from the rectangular shape of the LM-ROI of length $l$ and width $w$. Then, we take into account the (known) ratio $r$ between the pixel sizes of the EM and LM images. More specifically, we conduct two searches with two orientations of LoG EM patches (i.e., with a very coarse quantization of the rotation angle at this stage, but easy to implement): horizontal rectangle of dimensions $r l \times r w$ and vertical rectangle of dimensions $r w \times r l$ (i.e., after a $\pi / 2$ rotation). The selected patch will be the optimal one over the two searches.

\subsection{Registration of LM-EM patches}

The final selection of the LoG EM patch most similar to the LoG-LM-ROI also acts as a "pre-registration" step. Indeed, it provides with a combination of shift given by the difference in location of the LM-ROI and the selected LoG EM patch, magnification corresponding to the ratio between the EM patch size and the LM-ROI one, and possibly a $\pi / 2$ rotation if the type (vertical/horizontal) of the selected LoG EM patch is different than the ROI one. After decimating the LoG EM patch and warping it onto the LoG LM-ROI with this combined transformation, we further estimate a $2 \mathrm{D}$ affine motion model to refine the registration between the LoG EM patch and the LoG LM-ROI. Since the residual affine motion is likely to be small, we employ the Motion2D software ${ }^{1}$, implementing the robust gradient-based method described in [12]. Then, using the estimated transformation, we overlay the whole EM image onto the LM image around the ROI area.

\section{Experimental Results}

We tested our method on two sets of LM and EM images acquired at Institut Curie ${ }^{2}$. The LM image stacks (we will process a $2 \mathrm{D}$ projection) are acquired with epifluorescence Nikon Te2000, in five different channels, including transmitted illumination. We use the latter since it contains the most exploitable information to correlate with the EM image content. The section was then post-stained with Lead Citrate and imaged by a Tecnai Spirit FEI transmission electron microscopy (TEM) [7]. In the first experiment, the studied cell

\footnotetext{
${ }^{1}$ http://www.irisa.fr/vista/Motion2D/

${ }^{2}$ We thank X. Heiligenstein and P. Paul-Gilloteaux from UMR 144, Institut Curie, Paris, for providing us the images and the associated explanations.
}

elements are melanosomes, which are dark, dense biological material stored in round bags, visible both in transmitted light and EM. The LM image depicts several cells while the EM image is focused on the cell of interest. In the second experiment, the subcellular elements of interest are endosomes. The LM image contains several cells. The image pairs are displayed in Fig.1. We have framed the LM-ROI in blue and the ground-truth EM patch in red, both being defined by a biologist. The difference between LM and EM image contents is remarkable, both in appareance and size.

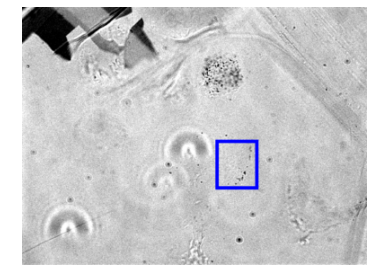

(a) LM1

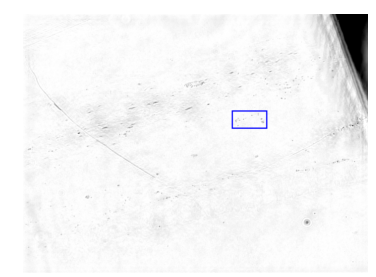

(c) LM2

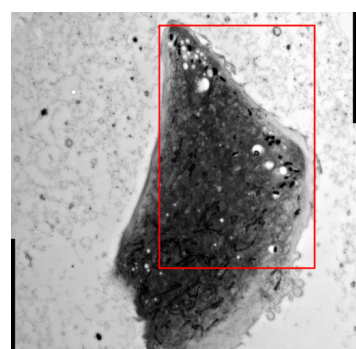

(b) EM1

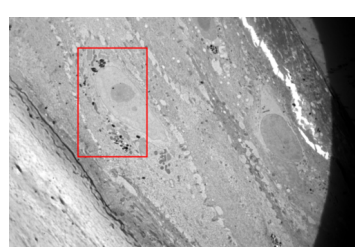

(d) EM2
Fig. 1: Input LM and EM images for experiment 1 (top) and experiment 2 (bottom) with ROI and ground-truth EM patch.

As explained above, using ATLAS we get the appropriate scale for the LoG transform of the EM and LM images. For the first experiment, they are respectively estimated as $s_{E M}=8.91$ and $s_{L M}=2.48$. For the second one, $s_{E M}=6.19$ and $s_{L M}=1.44$. The LoG representation of the EM and LM images are shown in Fig.2. As it can be observed, the appearance difference between the EM and LM images is significantly reduced. All the image contents will be better appreciated by zooming in the figures in the pdf file.

The first experiment involves a $512 \times 383 \mathrm{LM}$ image and a $4056 \times 3970$ EM image. Here, we designed the EM patch shape by hand. In the retracing stage, we exploit the LoGvalue histograms and the cosine distance. Similar results were obtained with the histogram intersection. We first tested EM patch locations at intervals of $2.3 \%$ of the $x$-dimension and $3.6 \%$ of the $y$-dimension (with $95 \%$ overlap). Then, we refined the search around the primarily selected location. By comparing Fig. $1 b$ and $2 b$, we can visually state that the EM patch location was correctly retrieved. The distance between the center of the ground-truth patch and the finally selected one is 66 pixels (i.e., $1.18 \%$ of the image diagonal).

The second experiment involves a $1392 \times 1040$ LM im- 
age and a $4008 \times 2664$ EM image, and the whole pipeline is fully automated. We used the LDP histograms, since the image content is less salient. We report results obtained with the histogram intersection. As the LM pixel size is $110 \mathrm{~nm}^{2}$, while the EM one is $11.3 \mathrm{~nm}^{2}$, we set $r=9.7$ to define the EM patch dimensions. We proceed with a first exhaustive search using intervals of $1.7 \%$ of the $x$-dimension and of $5.1 \%$ of the $y$-dimension (with $90 \%$ overlap), followed again by a search refinement. A quite satisfactory retracing is obtained, as visually assessed by comparing Fig.1d and $2 \mathrm{~d}$. The selected EM patch is vertical as expected. The distance between the ground-truth patch center and the finally selected one is 17 pixels $(0.35 \%$ of the image diagonal).

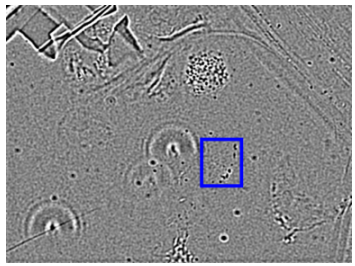

(a) LoG-LM1 and ROI

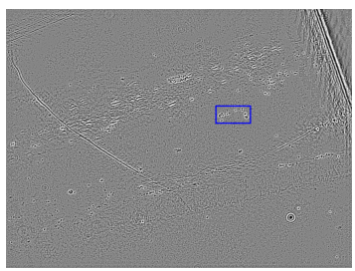

(c) LoG-LM2 and ROI

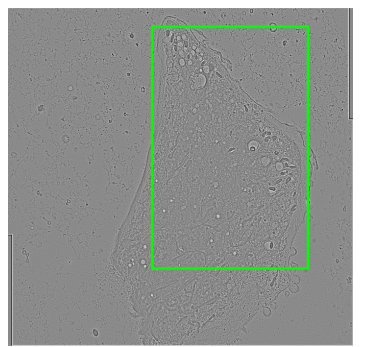

(b) LoG-EM1 and patch.

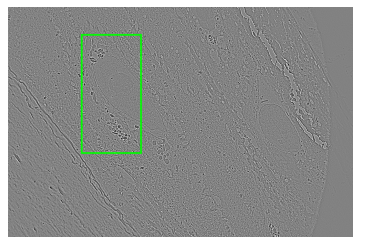

(d) LoG-EM2 and patch.
Fig. 2: LoG transform of LM and EM images with the LMROI in blue and the selected EM patch framed in green, experiment 1 (top row) and experiment 2 (bottom row).

The output of the search stage can be used to initialize the registration stage, where we estimate an affine motion model which accounts for both shift, rotation, scale and stretching. The estimated affine parameters are used to map the whole EM image (after decimation) over the LM image around the ROI area, and we come up with a rather accurate overlay as illustrated in Fig.3. The accuracy can be appreciated (using magnification in the pdf file) through the close alignment between corresponding EM and LM dots.

\section{Conclusion}

We have defined a fully automated method for the retracing and registration stages in the CLEM framework, which is a very challenging task due the great differences in appearance, size and field of view between LM and EM images. To make matching manageable, we introduced a common representation supplied by the LoG transform. To be invariant to size, scaling and rotation, we adopted normalized histograms
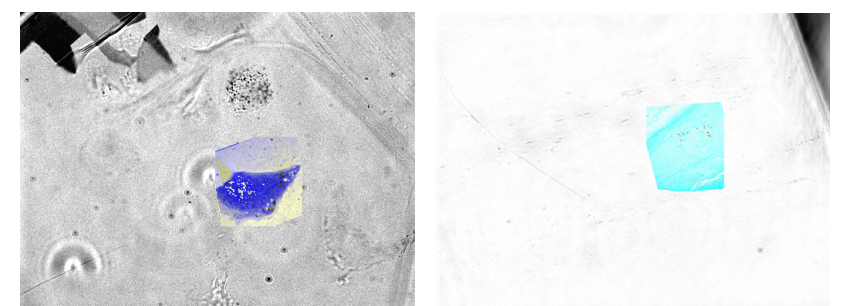

Fig. 3: Overlay of the whole (decimated and colorized) EM image onto the LM image for exp.1 (left), and exp.2 (right).

of LoG values or LDP values, in the retracing step. We reported convincing results on two real CLEM image sets. It yields a first validation of this novel approach. Future work will address the setting of a more comprehensive evaluation and a further improvement of the registration stage.

\section{References}

[1] A. Basset, J. Boulanger, J. Salamero, P. Bouthemy, and C. Kervrann. Adaptive spot detection with optimal scale selection in fluorescence microscopy images. IEEE Trans. Im. Proc., 24(11):4512-4527, 2015.

[2] P. de Boer, J.P. Hoogenboom, and B.N.G. Gipemans. Correlated light and electron microscopy: Ultastructure lights up! Nature Methods, 12(6):503-513, June 2015.

[3] T. Cao, C. Zach, S. Modla, D. Powell, K. Czymmek, and M. Niethammer. Multi-modal registration for correlative microscopy using image analogies. Medical Image Analysis, 18(6):914-926, 2014.

[4] S.-H. Cha. Comprehensive survey on distance/similarity measures between probability density functions. Int. J. of Math. Models and Methods in Applied Sciences, 1(4):300-307, 2007.

[5] B. N. Giepmans. Bridging fluorescence microscopy and electron microscopy. Histochemistry and Cell Biology, 130(2), 211-217, 2008.

[6] S. Handschuh, N. Baeumler, T. Schwaha, and B. Ruthensteiner. A correlative approach for combining microCT, light and transmission electron microscopy in a single 3D scenario. Front. Zool., 10(1):44, 2013.

[7] X. Heiligenstein et al. The CryoCapsule: Simplifying correlative light to electron microscopy. Traffic, 15(6):700-716, 2014.

[8] L. Hodgson, D. Nam, J. Mantell, A. Achim, and P. Verkade. Retracing in correlative light electron microscopy: where is my object of interest? Methods Cell Biol., 124:1-21, 2014.

[9] T. Jabid, M. H. Kabir, and O. Chae. Facial expression recognition using Local Directional Pattern (LDP). In ICIP, 2010.

[10] W. Kukulski at al. Precise, correlated fluorescence microscopy and electron tomography of lowicryl sections using fluorescent fiducial markers. Methods Cell Biol., 111:235-257, 2012.

[11] D. Nam, J. Mantell, L. Hodgson, D. Bull, P. Verkade, and A. Achim. Feature-based registration for correlative light and electron microscopy images. In ICIP, 2014.

[12] J.-M. Odobez and P. Bouthemy. Robust multiresolution estimation of parametric motion models. J. of Vis. Com. Im. Repr., 6:348-365, 1995.

[13] M. Perkovic et al. Correlative light- and electron microscopy with chemical tags. Journal of Structural Biology, 186(2):205-213, 2014.

[14] A. Sartori et al. Correlative microscopy: bridging the gap between fluorescence light microscopy and cryo-electron tomography. J. Struct. Biol., 160(2):135-145, Nov. 2007.

[15] C. Spiegelhalter, J.F. Laporte, and Y. Schwab. Correlative light and electron microscopy: from live cell dynamic to 3D ultrastructure. Methods in Molecular Biology, vol.1117, Chap.21, 484-501, Springer, 2014. 